\title{
The first 1000 days of life: traffic-related air pollution and development of wheezing and asthma in childhood. A systematic review of birth cohort studies
}

\author{
Alessandra Bettiol ${ }^{1}$, Elena Gelain ${ }^{2}$, Erika Milanesio ${ }^{3}$, Federica Asta $^{4}$ and Franca Rusconi ${ }^{5^{*}}$ (1)
}

\begin{abstract}
Background: The first 1000 days of life -including pregnancy and the first 2 years after birth- represent a critical window for health interventions.

This systematic review aimed to summarize the evidence on the relationship between traffic-related air pollutants exposure in the first 1000 days of life and the development of wheezing and asthma, with a particular focus on windows of exposure.
\end{abstract}

Methods: Medline and Embase were searched from January 2000 to May 2020 to retrieve population-based birthcohort studies, including registries, providing quantitative information on the association between exposure to traffic-related air pollutants during pregnancy or early life, and the risk of developing wheezing and asthma in childhood. Screening and selection of the articles were completed independently by three reviewers. The quality of studies was assessed using the Newcastle-Ottawa scale.

Results: Out of 9681 records retrieved, 26 studies from 21 cohorts were included. The most common traffic-related air pollutant markers were particulate matter (PM) and nitric oxides (NOx). The variability in terms of pollutants, exposure assessment methods, and exposure levels chosen to present the results did not allow a meta-analysis. Exposure to PM and NOx in pregnancy (10 cohorts) was consistently associated with an increased risk of asthma development, while the association with wheezing development was unclear. The second trimester of pregnancy seemed to be particularly critical for asthma risk. As for exposure during early life (15 cohorts), most studies found a positive association between PM (7/10 studies) and NOx (11/13 studies) and the risk of asthma development, while the risk of wheezing development was controversial. The period of postnatal exposure, however, was less precisely defined and a partial overlap between the period of exposure measurement and that of outcome development was present in a consistent number of studies (14 out of 15) raising doubts on the associations found.

(Continued on next page)

\footnotetext{
* Correspondence: franca.rusconi@meyer.it

${ }^{5}$ Unit of Epidemiology, Meyer Children's University Hospital, Viale Pieraccini

24, 50139 Florence, Italy

Full list of author information is available at the end of the article
}

C C The Author(s). 2021 Open Access This article is licensed under a Creative Commons Attribution 4.0 International License, which permits use, sharing, adaptation, distribution and reproduction in any medium or format, as long as you give appropriate credit to the original author(s) and the source, provide a link to the Creative Commons licence, and indicate if changes were made. The images or other third party material in this article are included in the article's Creative Commons licence, unless indicated otherwise in a credit line to the material. If material is not included in the article's Creative Commons licence and your intended use is not permitted by statutory regulation or exceeds the permitted use, you will need to obtain permission directly from the copyright holder. To view a copy of this licence, visit http://creativecommons.org/licenses/by/4.0/. The Creative Commons Public Domain Dedication waiver (http://creativecommons.org/publicdomain/zero/1.0/) applies to the data made available in this article, unless otherwise stated in a credit line to the data. 


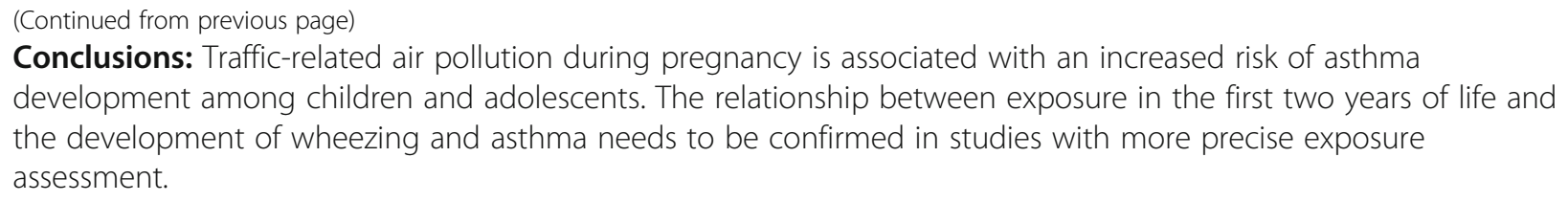

Keywords: Air pollution, Asthma, Children, Cohort studies, Early life, Pregnancy, Wheezing

\section{Background}

The period from conception to the child's second year of life (the first 1000 days) is a window for intervention to improve child and adult health [1]. This has been suggested for different exposures and outcomes, especially in the field of nutrition, cognitive development, and respiratory health $[2,3]$. Several programmes have therefore been undertaken worldwide with the aim of promoting early life interventions for children and families $[1,4]$.

Among early risk factors critical for respiratory health, tobacco smoke exposure, especially during pregnancy and in the first months after birth, is well known to be associated with an abnormal lung development and with an increased risk of both wheezing and asthma in offspring $[5,6]$. In fact, although lung growth occurs from conception to early adulthood, prenatal and early postnatal periods might be particularly vulnerable time windows [7].

Tobacco smoke and air pollution exposures are not equivalent, but air pollution exposure might have similar consequences for the lungs [7]. The advent of new technologies with a detailed assessment of exposure to air pollutants and a more precise spatial resolution allows nowadays to better explore the association between exposure to air pollutants from conception through infancy and respiratory outcomes later in life. Prospective birth cohorts represent the best design to assess the temporal relationship between early life exposures and the onset of respiratory diseases in childhood.

To date only one systematic review considering birth cohort studies published until March 2014 has focused on the relationship between childhood traffic-related air pollution exposure and subsequent asthma, wheeze, and allergic diseases [8]. Among the 11 cohort studies included in this systematic review [8], eight were population-based, while three were high-risk cohorts (i.e. including only subjects with a family history of asthma or allergies). Furthermore, almost all studies evaluated postnatal exposure, as studies on pregnancy exposure have been published later.

Since 2014, several birth cohort studies have focused on the association between exposure to traffic-related air pollutants, including gases - in particular nitrogen oxides $\left(\mathrm{NO}_{\mathrm{X}}\right)$ - and particulate matter $(\mathrm{PM})$ in pregnancy and in the first 2 years after birth and development of respiratory problems in childhood, namely wheezing and asthma.

On these bases, we aimed to systematically review the evidence from population-based birth cohort studies on the relationship between traffic-related air pollutants exposure in utero and in the first 2 years after birth (the first 1000 days of life) and the subsequent development of wheezing and asthma in childhood, with a particular focus on the critical time windows of exposure. A precise identification of the more vulnerable periods of exposure would be important to choose more efficacious preventive measures.

\section{Methods}

We searched Medline and Embase for papers published in English between January 1st 2000 and May 5th 2020.

We considered as eligible only prospective unselected pregnancy or birth cohort studies, including populationbased registries, providing quantitative information on the association between exposure to traffic-related air pollutants during pregnancy or during the first 2 years of infant's life, and the risk of developing wheezing and/ or asthma in children and adolescents (aged 1 to 17 years). Cohorts of susceptible populations, such as offspring of parents with asthma and/or allergies, were excluded. We considered exposures to any established traffic-related air pollutant, including black carbon (BC), carbon monoxide (CO), elemental carbon (EC), NOx, nitric oxide (NO), nitrogen dioxide $\left(\mathrm{NO}_{2}\right)$, hydrocarbons, and PM such as Ultra-Fine Particles $\leq 0.1 \mu \mathrm{m}$ in diameter (UFPs), $\mathrm{PM}<2.5$ and $<10 \mu \mathrm{m}$ in diameter $\left(\mathrm{PM}_{2.5}\right.$, $\mathrm{PM}_{10}$ ), PM between 2.5 and $10 \mu \mathrm{m}$ in diameter (PM coarse), and soot (i.e., black substance formed by combustion or separated from fuel during combustion, rising in fine particles). We excluded studies that: a) were reviews, commentaries, governmental reports, letters, animal and experimental studies; b) only examined adulthood asthma; c) only examined non-traffic-related air pollutants including ozone $\left(\mathrm{O}_{3}\right)$ which is not emitted directly from automobiles, sulphur dioxide $\left(\mathrm{SO}_{2}\right)$, indoor air pollution, proximity to point sources and wood smoke; d) only examined the association between the exposure to the selected pollutants and asthma exacerbations or severity; e) did not report the estimates of the 
quantitative association between traffic-related air pollutants and wheezing or asthma development.

The strategies used for Medline and Embase literature search are reported in supplementary Table 1. Briefly, search terms related to the three main thematic areas "traffic-related air pollutants", "wheezing/asthma" and "paediatric population" were combined through the Boolean operator "AND".

Titles and abstracts of all records retrieved by the search were screened by three co-authors (AB, EG, EM). We retrieved the full-text and supplementary material of all articles initially identified for potential inclusion. All potentially relevant full texts were independently screened by two pairs of co-authors to check the fulfilment of the inclusion criteria. Discrepancies were resolved through discussion.

In addition, we checked the reference list of previous published systematic reviews on this topic, to identify additional original research papers not retrieved by our search.To avoid study duplication, the following rules were adopted: a) where multiple publications were based on the same birth cohort or registry and considered the same exposures and outcomes within the same children's age group, only the most recent publication was included; b) where multiple publications were based on the same birth cohort or registry and evaluated the same exposures and respiratory outcomes for different age groups, we selected the publication with the earliest period of wheezing assessment, and the latest period of asthma assessment. The rationale for this choice was that wheezing occurring in the first years of life could have a different meaning in terms of prognosis with respect to wheezing and asthma at older ages and that asthma can be hardly diagnosed in the earlies years of life.

Data were extracted using a standardized form. Two authors $(\mathrm{AB}, \mathrm{EG})$ independently extracted the following data:

1. Exposure data: traffic-related air pollutants studied; mean or median or interquartile range (IQR) concentrations; period of exposure; method for exposure assessment.

2. Outcome data: outcome definition; method used to assess the outcome; period of outcome assessment; relevant adjusted effect estimates and 95\% Confidence Intervals (CI).

3. Other information: study population; year of publication; sample size; country in which participants were recruited.

The methodological quality of the studies was assessed by two authors (EM and $\mathrm{AB}$ ) using the NewcastleOttawa Quality Assessment Scale for cohort studies [9].

\section{Results}

Our search yielded to 9738 records. After removing duplicates, 9681 unique articles were identified. Of them, 9609 records were excluded after title and abstract screening, whereas 72 articles were selected for full-text reviewing. Among these, 26 articles [10-35] fulfilled the inclusion criteria (Fig. 1).

The 26 articles included in the review were based on 21 pregnancy or birth cohorts.

Nine birth cohorts were registry-based [14-17, 20, 21, $27,28,34,35]$. Two studies were case-control study, nested in a registry-based birth cohort $[21,35]$.

Ten cohorts were based in Europe [11, 13, 22-26, 29$34]$ and eight were based in North America [14, 16-21, $27,28,35]$. Of the remaining three cohorts, two were based in Asiatic countries [10,15] and one in Mexico [12]. Only four birth cohorts reported exposure to air pollutants both in pregnancy and in the first 2 years after delivery [13, 15-17].

The association between exposure to traffic-related air pollutants during pregnancy and the first 2 years of the child's life and subsequent asthma was evaluated in six [14-21] and 13 cohorts [15-17, 22, 23, 27-35], respectively (Tables 1 and 2).

Wheezing development was evaluated in nine cohorts: four after exposure in utero [10-13] and five after exposure in the first 24 months of child's life [13, 22-25] (Tables 3 and 4).

A large variability in the air pollutants studied and in the methods of exposure assessment was observed across studies. (supplementary Tables 2 and 3) The most common traffic-related air pollutant markers were PM $\left(\mathrm{PM}_{10}, \mathrm{PM}_{2.5}, \mathrm{PM}\right.$ coarse, and $\mathrm{PM}_{2.5}$ abs $)$ and $\mathrm{NO}_{2 .} \mathrm{A}$ few studies considered also other pollutants: $\mathrm{NOx}$, $\mathrm{NO}_{3}{ }^{-}, \mathrm{CO}$ and UFPs.

We observed a moderate variability in the methods for exposure assessment among studies that considered PM; most of the studies published in the last 5 years used models based on satellite data with a spatial resolution of $1-\mathrm{km}^{2}$, considering a complex and flexible modelling approach (supplementary Tables 2 and 3). For less recent studies on PM and for most of the studies on $\mathrm{NO}_{2}$ the most common method for exposure assessment was Land use regression (LUR) model. One study assessed exposure to $\mathrm{NO}_{3}{ }^{-}$in pregnancy using a hybrid model of a chemical transport model (GEOS-Chem) and land-use regression [19]. Two studies during pregnancy $[17,21]$ and eight in the first 2 years after delivery [17, 22-24, 26, 33-35] studied exposure to NOx, NO, CO, BC, soot and EC attributed to traffic (ECAT) applying different methods for exposure assessment (supplementary Tables 2 and 3).

The variability in terms of pollutants, exposure assessment methods, and exposure levels chosen to present 


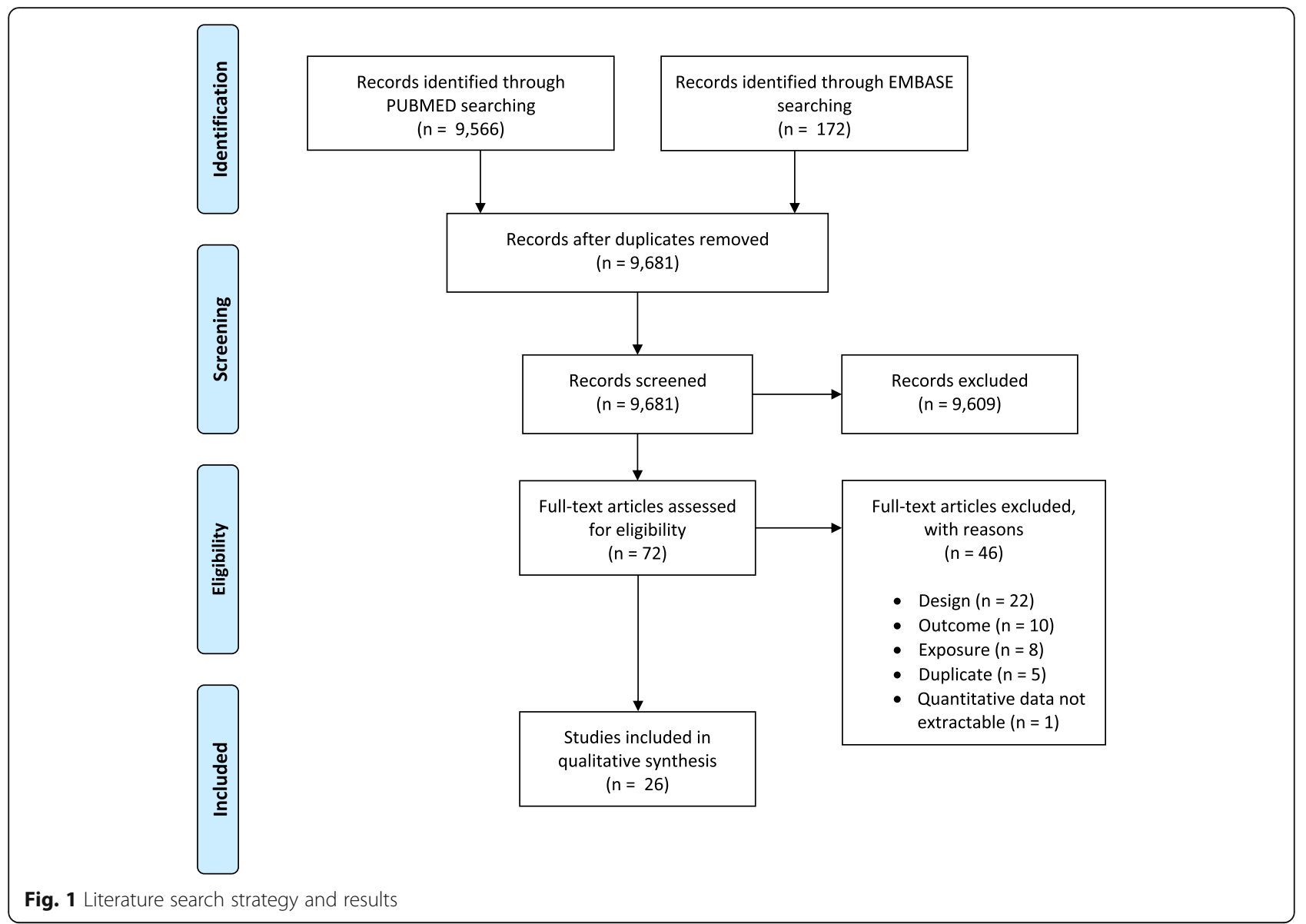

the results (e.g. interquartile range increase, mean or median levels etc.) as reported in detail in supplementary Tables 2 and 3 did not allow to do a meta-analysis.

Data on study quality are presented in supplementary Tables 4 and 5 .

Regarding the "Selection" items, all the studied cohorts were considered representative of the general population, as cohorts of susceptible populations were excluded.

In cohorts evaluating exposure in pregnancy the outcome of interest (wheezing or asthma in offspring) was, by definition, not present at the beginning of the study. Conversely, in all except one study [13, 15, 16, 22-35] which evaluated exposures in early life, there was an

Table 1 Association between exposure to traffic related air pollutants in pregnancy and wheezing development

\begin{tabular}{|c|c|c|c|c|c|c|}
\hline References & Type of study, country & $\begin{array}{l}\text { Subjects, } \\
\text { no }\end{array}$ & $\begin{array}{l}\text { Pollutants and exposure } \\
\text { assessment }\end{array}$ & Outcome & $\begin{array}{l}\text { Positive association } \\
\text { with the outcome }\end{array}$ & $\begin{array}{l}\text { Sensitivity } \\
\text { windows }\end{array}$ \\
\hline $\begin{array}{l}\text { Soh S et al., } \\
2018[10]\end{array}$ & $\begin{array}{l}\text { GUSTO birth cohort, } \\
\text { Singapore }\end{array}$ & 953 & $\begin{array}{l}\mathrm{PM}_{2.5} \\
\text { Daily exposure }\end{array}$ & $\begin{array}{l}\text { Wheezing } \\
\text { (birth up to } 2 \\
\text { years) }\end{array}$ & Yes & $\begin{array}{l}\text { No sensitive } \\
\text { trimester }\end{array}$ \\
\hline $\begin{array}{l}\text { Madsen C } \\
\text { et al., } 2017 \\
{[11]}\end{array}$ & $\begin{array}{l}\text { MoBa, pregnancy cohort, } \\
\text { Norway }\end{array}$ & 17,533 & $\begin{array}{l}\mathrm{NO}_{2} \\
\text { Annual average estimates at } \\
\text { residential address at birth }\end{array}$ & $\begin{array}{l}\text { Wheezing } \\
\text { (6 to } 18 \\
\text { months) }\end{array}$ & $\mathrm{No}$ & NA \\
\hline $\begin{array}{l}\text { Rosa MJ et al., } \\
2017 \text { [12] }\end{array}$ & $\begin{array}{l}\text { PROGRESS pregnancy } \\
\text { cohort, Mexico }\end{array}$ & 552 & $\begin{array}{l}\mathrm{PM}_{2.5} \\
\text { Daily exposure }\end{array}$ & $\begin{array}{l}\text { Wheezing } \\
\text { (birth up to } 4 \\
\text { years) }\end{array}$ & No & $\begin{array}{l}\text { No sensitive } \\
\text { trimester }\end{array}$ \\
\hline $\begin{array}{l}\text { Aguilera I } \\
\text { et al., } 2013 \\
{[13]}\end{array}$ & $\begin{array}{l}\text { Four birth cohorts of the } \\
\text { INMA project, Spain }\end{array}$ & 2199 & $\begin{array}{l}\mathrm{NO}_{2} \\
\text { Annual average estimates at } \\
\text { residential address in pregnancy }\end{array}$ & $\begin{array}{l}\text { Wheezing } \\
\text { (birth up to } \\
12-18 \text { months) }\end{array}$ & $\mathrm{No}$ & NA \\
\hline
\end{tabular}

$P M_{2.5}$ Particulate matter $<2.5 \mu \mathrm{m}$ in diameter; $\mathrm{NO}_{2}$ Nitrogen dioxide, NA Not assessed 
Table 2 Association between exposure to traffic related air pollutants in pregnancy and asthma development

\begin{tabular}{|c|c|c|c|c|c|c|}
\hline References & $\begin{array}{l}\text { Type of study, } \\
\text { Country }\end{array}$ & Subjects, no & $\begin{array}{l}\text { Pollutants and } \\
\text { exposure } \\
\text { assessment }\end{array}$ & Outcome & $\begin{array}{l}\text { Positive } \\
\text { association with } \\
\text { the outcome }\end{array}$ & $\begin{array}{l}\text { Sensitivity } \\
\text { windows }\end{array}$ \\
\hline $\begin{array}{l}\text { Lavigne E } \\
\text { et al., } 2019 \\
\text { [14] }\end{array}$ & $\begin{array}{l}\text { Registry-based birth cohort, } \\
\text { Toronto, Canada }\end{array}$ & 160,641 & $\begin{array}{l}\text { UFPs, } \mathrm{PM}_{2.5 \prime} \\
\mathrm{NO}_{2} \\
\text { Daily exposure }\end{array}$ & $\begin{array}{l}\text { Asthma } \\
\text { (birth up to } \\
<6 \text { years) }\end{array}$ & Yes & $\begin{array}{l}\text { Second } \\
\text { trimester }\end{array}$ \\
\hline $\begin{array}{l}\text { Jung CR } \\
\text { et al., } 2019 \\
{[15]}\end{array}$ & $\begin{array}{l}\text { TMCHD registry-based birth } \\
\text { cohort, Taiwan }\end{array}$ & 184,604 & $\begin{array}{l}\mathrm{PM}_{2.5} \\
\text { Daily exposure }\end{array}$ & $\begin{array}{l}\text { Asthma } \\
\text { (birth up to } \\
3-10 \text { years) }\end{array}$ & Yes & Weeks 6-22 \\
\hline $\begin{array}{l}\text { Lavigne E } \\
\text { et al., } 2018 \\
\text { [16] }\end{array}$ & $\begin{array}{l}\text { Registry-based birth cohort, } \\
\text { Ontario, Canada }\end{array}$ & 222,864 & $\begin{array}{l}\mathrm{PM}_{2.5}, \mathrm{NO}_{2} \\
\text { Daily exposure }\end{array}$ & $\begin{array}{l}\text { Asthma } \\
\text { (birth up to } \\
<6 \text { years) }\end{array}$ & Yes & $\begin{array}{l}\text { Second } \\
\text { trimester }\end{array}$ \\
\hline $\begin{array}{l}\text { Pennington } \\
\text { AF et al., } \\
2018 \text { [17] }\end{array}$ & $\begin{array}{l}\text { KAPPA, registry-based birth } \\
\text { cohort, Atlanta, USA }\end{array}$ & 19,951 & $\begin{array}{l}\mathrm{PM}_{2.5}, \mathrm{NO}_{x_{1}} \mathrm{CO} \\
\text { Annual average }\end{array}$ & $\begin{array}{l}\text { Asthma } \\
\text { ( } 2 \text { to } 6 \text { years) }\end{array}$ & Yes & NA \\
\hline $\begin{array}{l}\text { Lee A et al., } \\
2018[18]\end{array}$ & $\begin{array}{l}\text { ACCESS pregnancy cohort, } \\
\text { Boston, USA }\end{array}$ & 736 & $\begin{array}{l}\mathrm{PM}_{2.5} \\
\text { Daily exposure }\end{array}$ & $\begin{array}{l}\text { Asthma } \\
\text { (birth up to } \\
6 \text { years) }\end{array}$ & Yes & $\begin{array}{l}\text { Weeks } 19-23 \\
\text { in exposed to } \\
\text { maternal } \\
\text { prenatal stress }\end{array}$ \\
\hline $\begin{array}{l}\text { Bose S et al., } \\
2017[19]\end{array}$ & $\begin{array}{l}\text { ACCESS pregnancy cohort, } \\
\text { Boston, USA }\end{array}$ & 752 & $\begin{array}{l}\mathrm{NO}_{3}^{-} \\
\text {Daily exposure }\end{array}$ & $\begin{array}{l}\text { Asthma } \\
\text { (birth up to } \\
6 \text { years) }\end{array}$ & $\begin{array}{l}\text { Yes } \\
\text { in boys exposed } \\
\text { to prenatal } \\
\text { maternal stress }\end{array}$ & $\begin{array}{l}7-19 \text { and } 33- \\
40 \text { weeks }\end{array}$ \\
\hline $\begin{array}{l}\text { Sbihi H } \\
\text { et al., } 2017 \\
\text { [20] }\end{array}$ & $\begin{array}{l}\text { Registry-based birth cohort, } \\
\text { Vancouver, Canada }\end{array}$ & 65,254 & $\begin{array}{l}\mathrm{NO}_{2}, \mathrm{PM}_{2.5} \\
\text { Daily exposure } \\
\text { aggregated over the } \\
\text { pregnancy period }\end{array}$ & $\begin{array}{l}\text { Asthma } \\
\text { trajectories } \\
\text { (birth up to } \\
7-10 \text { years) }\end{array}$ & Yes & NA \\
\hline $\begin{array}{l}\text { Sbihi H } \\
\text { et al., } 2016 \\
\text { [21] }\end{array}$ & $\begin{array}{l}\text { Case-control nested in a } \\
\text { registry-based birth cohort, } \\
\text { Vancouver, Canada }\end{array}$ & $\begin{array}{l}\text { Pre-schoolers: } 6948 \text { cases, } \\
34,621 \text { controls; School-age: } \\
1711 \text { and } 8577\end{array}$ & $\begin{array}{l}\mathrm{BC}, \mathrm{CO}, \mathrm{NO}, \mathrm{NO}_{2} \\
\mathrm{PM}_{2.5}, \mathrm{PM}_{10} \\
\text { Daily exposure } \\
\text { aggregated over the } \\
\text { pregnancy period }\end{array}$ & $\begin{array}{l}\text { Asthma } \\
\text { (birth up to } \\
6-10 \text { years) }\end{array}$ & $\begin{array}{l}\text { Yes } \\
\text { only in } \\
\text { preschoolers and } \\
\text { only for } \mathrm{PM}_{10}\end{array}$ & NA \\
\hline
\end{tabular}

$B C$ Black carbon, $C O$ Carbon monoxide, $P M_{2.5}$ Particulate matter $<2.5 \mu \mathrm{m}$ in diameter, $P M_{10}$ Particulate matter $<10 \mu \mathrm{m}$ in diameter; $N O_{x}$ Nitrogen oxides, $N O$ Nitric oxide, $\mathrm{NO}_{2}$ Nitrogen dioxide, $\mathrm{NO}_{3}$ Nitrate, UFPs Ultra-Fine Particles $\leq 0.1 \mu \mathrm{m}$ in diameter; $N A$ Not assessed

Table 3 Association between exposure to traffic related air pollutants in early life and wheezing development

\begin{tabular}{|c|c|c|c|c|c|}
\hline References & $\begin{array}{l}\text { Type of study, } \\
\text { Country }\end{array}$ & $\begin{array}{l}\text { Subjects, } \\
\text { no }\end{array}$ & Pollutants and exposure assessment & Outcome & $\begin{array}{l}\text { Positive association with the } \\
\text { outcome }\end{array}$ \\
\hline $\begin{array}{l}\text { Rancière F et al., } \\
2017 \text { [22] }\end{array}$ & $\begin{array}{l}\text { PARIS birth cohort, } \\
\text { France }\end{array}$ & 2015 & $\begin{array}{l}\mathrm{NO}_{x} \\
\text { Exposure assessed in the first year of } \\
\text { life }\end{array}$ & $\begin{array}{l}\text { Wheezing } \\
\text { phenotypes } \\
\text { (birth up to } 4 \\
\text { years) }\end{array}$ & $\begin{array}{l}\text { Yes } \\
\text { only for persistent wheezing }\end{array}$ \\
\hline $\begin{array}{l}\text { Aguilera I et al., } \\
2013 \text { [13] }\end{array}$ & $\begin{array}{l}\text { Four birth cohorts, } \\
\text { INMA project, Spain }\end{array}$ & 2199 & $\begin{array}{l}\mathrm{NO}_{2} \\
\text { Annual average exposure estimated at } \\
\text { address in the first year of life }\end{array}$ & $\begin{array}{l}\text { Wheezing } \\
\text { (birth up to } \\
12-18 \text { months) }\end{array}$ & No \\
\hline $\begin{array}{l}\text { Gehring U et al., } \\
2010 \text { [23] }\end{array}$ & $\begin{array}{l}\text { PIAMA birth cohort, } \\
\text { the Netherlands }\end{array}$ & 3863 & $\begin{array}{l}\mathrm{PM}_{2.5}, \mathrm{NO}_{2} \text {, Soot } \\
\text { Annual average exposure estimated at } \\
\text { birth address }\end{array}$ & $\begin{array}{l}\text { Wheezing } \\
\text { phenotypes } \\
\text { (birth up to } 8 \\
\text { years) }\end{array}$ & $\begin{array}{l}\text { Yes } \\
\text { only for } \mathrm{PM}_{2.5} \text { and early transient } \\
\text { and late onset wheezing }\end{array}$ \\
\hline $\begin{array}{l}\text { Nordling E et al., } \\
2008 \text { [24] }\end{array}$ & Birth cohort, Sweden & 3515 & $\begin{array}{l}\mathrm{PM}_{10}, \mathrm{NO}_{x} \\
\text { Annual average exposure estimated at } \\
\text { address in the first year of life }\end{array}$ & $\begin{array}{l}\text { Wheezing } \\
\text { phenotypes } \\
\text { (birth up to } 4 \\
\text { years) }\end{array}$ & $\begin{array}{l}\text { Yes } \\
\text { only for } \mathrm{NO}_{x} \text { and persistent } \\
\text { wheezing. }\end{array}$ \\
\hline $\begin{array}{l}\text { Morgenstern V } \\
\text { et al., } 2007 \text { [25] }\end{array}$ & $\begin{array}{l}\text { GINI/LISA birth cohorts, } \\
\text { Munich, Germany }\end{array}$ & 3577 & $\begin{array}{l}\mathrm{PM}_{2.5} \text { mass, } \mathrm{PM}_{2.5} \text { absorbance, } \mathrm{NO}_{2} \\
\text { Annual average exposure estimated at } \\
\text { birth address }\end{array}$ & $\begin{array}{l}\text { Wheezing } \\
\text { (birth up to } 2 \\
\text { years) }\end{array}$ & No \\
\hline $\begin{array}{l}\text { Brauer M et al., } \\
2002[26]\end{array}$ & $\begin{array}{l}\text { PIAMA birth cohort, } \\
\text { the Netherlands }\end{array}$ & 3730 & $\begin{array}{l}\mathrm{PM}_{2.5}, \mathrm{NO}_{2} \text {, Soot } \\
\text { Annual average exposure estimated at } \\
\text { birth address }\end{array}$ & $\begin{array}{l}\text { Wheezing } \\
\text { (birth up to } 2 \\
\text { years) }\end{array}$ & No \\
\hline
\end{tabular}


Table 4 Association between exposure to traffic related air pollutants in early life and asthma development

\begin{tabular}{|c|c|c|c|c|c|}
\hline References & $\begin{array}{l}\text { Type of study, } \\
\text { Country }\end{array}$ & $\begin{array}{l}\text { Subjects, } \\
\text { no }\end{array}$ & $\begin{array}{l}\text { Pollutant and exposure } \\
\text { assessment }\end{array}$ & Outcome & $\begin{array}{l}\text { Positive association } \\
\text { with the outcome }\end{array}$ \\
\hline $\begin{array}{l}\text { To T et al., } \\
2020[27]\end{array}$ & $\begin{array}{l}\text { T-CHEQ registry-based birth cohort, On- } \\
\text { tario, Canada }\end{array}$ & 1286 & $\begin{array}{l}\mathrm{PM}_{2.5}, \mathrm{NO}_{2} \\
\text { Average exposure assessed in the } \\
\text { first } 3 \text { years of life }\end{array}$ & $\begin{array}{l}\text { Asthma } \\
\text { (birth up to } \\
\text { 15-20 years) }\end{array}$ & $\begin{array}{l}\text { Yes } \\
\text { only for } \mathrm{NO}_{2}\end{array}$ \\
\hline $\begin{array}{l}\text { Jung CR et al., } \\
2019[15]\end{array}$ & $\begin{array}{l}\text { TMCHD registry-based birth cohort, } \\
\text { Taiwan }\end{array}$ & 184,604 & $\begin{array}{l}\mathrm{PM}_{2.5} \\
\text { Exposure assessed in the first year of } \\
\text { life }\end{array}$ & $\begin{array}{l}\text { Asthma } \\
\text { (birth up to } \\
3-10 \text { years) }\end{array}$ & Yes \\
\hline $\begin{array}{l}\text { Lavigne E } \\
\text { et al. } 2018 \\
{[16]}\end{array}$ & $\begin{array}{l}\text { Registry-based birth cohort Ontario, } \\
\text { Canada }\end{array}$ & 222,864 & $\begin{array}{l}\mathrm{PM}_{2.5}, \mathrm{NO}_{2} \\
\text { Exposure assessed in the first year of } \\
\text { life }\end{array}$ & $\begin{array}{l}\text { Asthma } \\
\text { (birth up to } \\
<6 \text { years) }\end{array}$ & $\begin{array}{l}\text { Yes } \\
\text { only for } \mathrm{NO}_{2}\end{array}$ \\
\hline $\begin{array}{l}\text { Pennington } \\
\text { AF et al., } 2018 \\
{[17]}\end{array}$ & $\begin{array}{l}\text { KAPPA registry- based birth cohort, } \\
\text { Atlanta, USA }\end{array}$ & 23,100 & $\begin{array}{l}\mathrm{PM}_{2.5} \mathrm{NO}_{x_{1}} \mathrm{CO} \\
\text { Annual average exposure estimated } \\
\text { in the first year of life }\end{array}$ & $\begin{array}{l}\text { Asthma } \\
(2 \text { to } 6 \\
\text { years })\end{array}$ & Yes \\
\hline $\begin{array}{l}\text { Rancière F } \\
\text { et al., } 2017 \\
{[22]}\end{array}$ & PARIS birth cohort, France & 2015 & $\begin{array}{l}\text { NOx } \\
\text { Exposure assessed in the first year of } \\
\text { life }\end{array}$ & $\begin{array}{l}\text { Asthma } \\
\text { (birth up to } \\
4 \text { years) }\end{array}$ & Yes \\
\hline $\begin{array}{l}\text { Tétreault L-F } \\
\text { et al., } 2016 \\
{[28]}\end{array}$ & $\begin{array}{l}\text { Registry-based birth cohort, Quebec, } \\
\text { Canada }\end{array}$ & $1,183,865$ & $\begin{array}{l}\mathrm{PM}_{2.5}, \mathrm{NO}_{2} \\
\text { Annual average exposure estimated } \\
\text { at birth address }\end{array}$ & $\begin{array}{l}\text { Asthma } \\
\text { (birth up to } \\
1-12 \text { years) }\end{array}$ & Yes \\
\hline $\begin{array}{l}\text { Gehring } U \\
\text { et al., } 2015 \\
{[30]}\end{array}$ & PIAMA birth cohort, the Netherlands & 3702 & $\begin{array}{l}\mathrm{PM}_{2.5} \text { abs, } \mathrm{PM}_{2.5}, \mathrm{PM}_{10}, \mathrm{PM} \text { coarse, } \\
\mathrm{NO}_{2} \text {, elemental composition of } \mathrm{PM}_{2.5} \\
\text { and } \mathrm{PM}_{10} \\
\text { Annual average exposure estimated } \\
\text { at birth address }\end{array}$ & $\begin{array}{l}\text { Asthma } \\
\text { (birth up to } \\
11 \text { years) }\end{array}$ & $\begin{array}{l}\text { Yes } \\
\text { only for } \mathrm{NO}_{2}, \mathrm{~K} \mathrm{PM}_{2.5} \\
\mathrm{KPM}_{10}, \mathrm{SPM}_{2.5}, \mathrm{Zn} \\
\mathrm{PM}_{10}\end{array}$ \\
\hline $\begin{array}{l}\text { Gehring } U \\
\text { et al., } 2015 \\
{[29]}\end{array}$ & $\begin{array}{l}\text { BAMBSE, GINI plus, LISA plus and PIAMA } \\
\text { birth cohorts, Sweden, Germany, the } \\
\text { Netherlands }\end{array}$ & 14,126 & $\begin{array}{l}\mathrm{PM}_{2.5} \text { abs, } \mathrm{PM}_{2.5}, \mathrm{PM}_{10}, \mathrm{PM} \text { coarse, } \\
\mathrm{NO}_{2} \\
\text { Annual average exposure estimated } \\
\text { at birth address }\end{array}$ & $\begin{array}{l}\text { Asthma } \\
\text { (birth up to } \\
\text { 14-16 years) }\end{array}$ & $\begin{array}{l}\text { Yes } \\
\text { only for } \mathrm{NO}_{2}, \mathrm{PM}_{2.5} \\
\text { abs }\end{array}$ \\
\hline $\begin{array}{l}\text { Ranzi A et al., } \\
2014 \text { [31] }\end{array}$ & GASPII birth cohort, Italy & 672 & $\begin{array}{l}\mathrm{NO}_{2} \\
\text { Annual average exposure estimated } \\
\text { at birth address }\end{array}$ & $\begin{array}{l}\text { Asthma } \\
\text { (birth up to } \\
7 \text { years) }\end{array}$ & No \\
\hline $\begin{array}{l}\text { Fuertes E } \\
\text { et al., } 2013 \\
{[32]}\end{array}$ & $\begin{array}{l}\text { GINI plus and LISA plus birth cohorts, } \\
\text { Germany }\end{array}$ & 6604 & $\begin{array}{l}\mathrm{PM}_{2.5} \text { mass, } \mathrm{PM}_{2.5} \text { abs, } \mathrm{NO}_{2} \\
\text { Annual average exposure estimated } \\
\text { at birth address }\end{array}$ & $\begin{array}{l}\text { Asthma } \\
\text { (birth up to } \\
10 \text { years) }\end{array}$ & No \\
\hline $\begin{array}{l}\text { Gruzieva O } \\
\text { et al., } 2013 \\
\text { [33] }\end{array}$ & BAMSE birth cohort, Sweden & 3633 & $\begin{array}{l}\mathrm{NO}_{x,} \mathrm{PM}_{10} \\
\text { Annual average exposure estimated } \\
\text { at birth address }\end{array}$ & $\begin{array}{l}\text { Asthma } \\
\text { (birth up to } \\
12 \text { years) }\end{array}$ & Yes \\
\hline $\begin{array}{l}\text { Lindgren A } \\
\text { et al., } 2013 \\
\text { [34] }\end{array}$ & $\begin{array}{l}\text { Registry-based birth cohort, southern } \\
\text { Sweden }\end{array}$ & 7898 & $\begin{array}{l}\mathrm{NO}_{x} \\
\text { Annual average exposure estimated } \\
\text { at birth address }\end{array}$ & $\begin{array}{l}\text { Asthma } \\
\text { (birth up to } \\
1-6 \text { years) }\end{array}$ & Negative association \\
\hline $\begin{array}{l}\text { Clark NA } \\
\text { et al., } 2010 \\
{[35]}\end{array}$ & $\begin{array}{l}\text { Case-control study, nested in a cohort } \\
\text { (administrative databases), British } \\
\text { Columbia, Canada }\end{array}$ & $\begin{array}{l}3482 \text { cases, } \\
17,410 \\
\text { controls }\end{array}$ & $\begin{array}{l}\mathrm{PM}_{2.5}, \mathrm{PM}_{10}, \mathrm{NO}, \mathrm{NO}_{2}, \mathrm{CO}, \mathrm{BC} \\
\text { Exposure estimated at address in the } \\
\text { first year of life }\end{array}$ & $\begin{array}{l}\text { Asthma } \\
\text { (birth up to } \\
36-59 \\
\text { months) }\end{array}$ & $\begin{array}{l}\text { Yes } \\
\text { only for } \mathrm{NO}_{2}, \mathrm{BC}, \mathrm{CO} \text {, } \\
\mathrm{PM}_{10}\end{array}$ \\
\hline $\begin{array}{l}\text { Gehring } U \\
\text { et al., } 2010 \\
\text { [23] }\end{array}$ & $\begin{array}{l}\text { PIAMA birth cohort, } \\
\text { the Netherlands }\end{array}$ & 3863 & $\begin{array}{l}\mathrm{PM}_{2.5}, \mathrm{NO}_{2} \text {, Soot } \\
\text { Annual average exposure estimated } \\
\text { at birth address }\end{array}$ & $\begin{array}{l}\text { Asthma } \\
\text { (birth up to } \\
8 \text { years) }\end{array}$ & Yes \\
\hline
\end{tabular}

Abs Absorbance, $B C$ Black carbon, $C O$ Carbon monoxide, $P M_{2.5}$ Particulate matter $<2.5 \mu \mathrm{m}$ in diameter, $P M_{10}$ Particulate matter $<10 \mu \mathrm{m}$ in diameter, $N O_{x}$ Nitrogen oxides, NO Nitric oxide, $\mathrm{NO}_{2}$ Nitrogen dioxide, $\mathrm{NO}_{3}$ Nitrate, UFPs Ultra-Fine Particles $\leq 0.1 \mu \mathrm{m}$ in diameter, NA Not assessed

overlap between the period of exposure measurement and that of outcome development. This might represent a relevant risk of bias, especially for studies in which the outcome of interest was wheezing evaluated in the first few months/years of life.

Regarding the "Comparability" domain (supplementary Tables 4 and 5), except for five studies assessing in utero exposure [17-21] and two studies assessing exposure in early life $[17,28]$ all the other studies adjusted for both second-hand smoking and asthma predisposition, important potential confounders of the association between exposure to traffic-related air pollutants and wheezing and asthma. Five of 12 studies on pregnancy exposure to air pollutants adjusted for exposure during early life [10, $12,14-16]$ while only three of 16 studies on early life exposures also accounted for it in their analysis exposure 
during pregnancy $[15,16,35]$. Moreover, several cohorts considered - often in sensitivity analyses - also changes of home address for a more precise evaluation of exposure to air pollutants [11, 13-21, 23, 26-32, 34, 35].

As for the "Outcome" domain (supplementary Tables 4 and 5), we defined that a follow-up of 2 and of 6 years was long enough to detect the occurrence of wheezing and asthma, respectively. According to this definition, for exposure in pregnancy follow-up was not long enough for wheezing or asthma to occur in two [11, 13] and two cohorts $[15,17]$, respectively. For exposures in the first 2 years of life follow up was not long enough for wheezing to occur in all the subjects in one cohort [13] and for asthma in four cohorts [15, 17, 34, 35].

Only three cohorts had a follow-up rate $\leq 60 \%$, considered as likely to introduce a bias $[17,22,31]$.

Tables 1 and 2 and supplementary Table 2 provide a summary of the 12 studies evaluating the association between exposure to traffic-related air pollutants in pregnancy and wheezing and asthma development [10-21].

The sample sizes ranged from 552 to 222,864 , being the largest cohorts based on registries. Most of the studies evaluated exposures to particulate matter ( $9 / 12$ studies) and eight to gases including $\mathrm{NO}_{2}$ (six studies), $\mathrm{NOx}$, $\mathrm{NO}_{3}{ }^{-}, \mathrm{NO}$, and $\mathrm{CO}$.

Follow-up periods varied according to the outcome, ranging from 6 to 48 months for wheezing and from 2 to 10 years for asthma, though in the majority of studies on asthma incidence children were followed up at least up to school age.

Only 4 studies examined the development of wheezing after exposure to traffic-related air pollutants in pregnancy [10-13]. One study (GUSTO birth cohort, Singapore; 953 subjects) [10] reported an association between $\mathrm{PM}_{2.5}$ measured at eight stations and wheezing in the first 2 years of life. This was not confirmed in another small birth cohort (PROGRESS pregnancy cohort, Mexico; 552 subjects) [12]. No association was found for exposure to $\mathrm{NO}_{2}$ in pregnancy either in the INMA birth cohort in Spain (2199 subjects) [13] and in the MoBa pregnancy cohort in Norway [11]; this was a large cohort (17.533 subjects) exposed to low levels of $\mathrm{NO}_{2}$ (mean: $13.6 \mu \mathrm{g} / \mathrm{m} 3)$.

Conversely, a positive association between exposure to both particulate and gases during pregnancy and asthma development was found in all the studies.

Five studies tried to identify "sensitive time periods" for exposure to air pollutants during the prenatal period and asthma development [14-16, 18, 19]. A sensitive window was found in four studies $[14-16,18]$ in the second trimester of pregnancy (weeks 13 to 24) for exposures either to UFP, $\mathrm{PM}_{2.5}$, or to $\mathrm{NO}_{2}$. Notably, the susceptibility during this sensitive window seemed to be more critical for boys with elevated maternal stress during gestation [18].

Tables 3 and 4 and supplementary Table 3 describe the 19 studies [13, 15-17, 23-26, 28-35] evaluating the association between exposure to traffic-related pollutants in the first 2 years of children's life and wheezing and asthma development.

The sample sizes ranged from 672 to 1.183 .865 subjects. Seventeen studies evaluated exposures to gases and 14 to PM.

Follow-up periods varied according to the outcome, being from 12 months to 8 years for wheezing and from 12 months to 16 years for asthma.

Three studies that followed children up to 4-8 years of life focused on wheezing phenotypes (Table 3): two found an association between exposure to NOx and persistent wheezing $[22,24]$ and one between $\mathrm{PM}_{2.5}$ and early transient and late-onset wheezing [23]. No association was found in three studies that evaluated exposure to $\mathrm{NO}_{2}$ or $\mathrm{PM}_{2.5}$ and wheezing in the first 2 years of children's life with no mention of phenotypes $[13,25$, 26].

Eleven [15-17, 22, 23, 27-30, 33, 35] of 14 studies found an association with exposure to one or more pollutants at the birth address or in the first year(s) of life and development of asthma. (Table 4) A positive association with asthma incidence was found more often for $\mathrm{NO}_{2}$ and $\mathrm{PM}_{2.5}$. One study performed in Italy [31] on a small cohort (672 subjects) did not find an association between exposure to $\mathrm{NO}_{2}$ measured at the birth address and development of asthma in the first 7 years of life. A study in the GINA plus and LISA plus birth cohorts (6604 subjects) [32] also did not find an association between exposure to $\mathrm{PM}_{2.5}$ and $\mathrm{NO}_{2}$ at the birth address and asthma incidence from birth up to 10 years. However, in another study [29] where data from the same cohorts collected over a longer follow-up period (14 to 16 years) were put together to those of other larger cohorts (BAMSE and PIAMA) and meta-analyzed, an association was found for $\mathrm{NO}_{2}$ and $\mathrm{PM}_{2.5}$. Finally, Lindgren and colleagues [34] found a negative association between exposure to NOx at birth and the development of asthma in children aged 2 to 6 years, though the study, also according to authors, might have been subjected to several biases.

\section{Discussion}

Our systematic review summarized current published evidence from prospective unselected cohort studies on the association between exposure to traffic-related air pollutants in the first 1000 days of life -including pregnancy and the first 2 years after birth- and the subsequent risk of developing asthma and wheezing in childhood. We found consistent results for exposure to 
both NOx and PM in pregnancy and asthma development in childhood [14-21], with a more vulnerable window of exposure in the weeks corresponding to the second trimester of pregnancy $[14-16,18]$. The susceptibility during this window of exposure seems to be modified by gender and stress-related factors; in fact, air pollution exposure during thesecond trimester of pregnancy (weeks 19-23) seems more critical in case of elevated maternal stress during gestation, particularly for male newborns [18].

The relationship between exposure to air pollutants in pregnancy and development of wheezing in childhood was evaluated in only four studies [10-13], and a significant association was found with exposure to $\mathrm{PM}_{2.5}$ in only one [10], while two studies did not find an association with exposure to $\mathrm{NO}_{2}[11,13]$.

Also, for exposures to traffic-related air pollutants in the first 2 years after birth, the results were not concordant for wheezing development, while a positive association was found in most of the studies evaluating PM and NOx and the risk of asthma development [15-17, 23, 27-30, 33, 35].

As previously discussed, a large variability among studies in terms of pollutants considered, exposure assessment, and air pollutants levels, prevented us to perform a meta-analysis.

On the other hand, an accurate evaluation of the characteristics and the quality of the studies included in this systematic review gave interesting hints and allowed several important considerations.

The association found for exposure in pregnancy and asthma at school age is concordant with findings of an adverse impact of prenatal air pollution exposure on lung function [36-38]. In three studies [14, 16, 19] the second trimester of pregnancy was identified as a vulnerable period for asthma development both for exposure to $\mathrm{PM}$ and $\mathrm{NO}_{2}$. In studies evaluating lung function, the evidence of a more vulnerable trimester is weaker, though two studies also mentioned the second trimester $[38,39]$. A recent Editorial [40] on inconclusive results on the most vulnerable time-period of exposure in pregnancy for lung function outcome in childhood pointed out methodological issues, highlighting the need of a more precise exposure assessment and statistical methods able to identify weeks of gestation rather than specific trimesters. In four studies included in our review $[14-16,18]$ which identified the second trimester of pregnancy as a vulnerable period, daily exposures were available, and distributed lag nonlinear models were used to identify susceptible weeks, thus allowing a precise definition of time windows of exposure. The availability of only two studies based on small birth cohorts $[10,12]$ evaluating the association between intrauterine $\mathrm{PM}_{2.5}$ exposure and wheezing in offspring as the outcome, and which found opposite results, does not permit to derive any conclusion. Exposure to LURmodelled prenatal traffic-related $\mathrm{NO}_{2}$ was also evaluated in two larger birth cohorts $[11,13]$ and no association was found for the development of wheezing in the first 18 months of life. Mean $\mathrm{NO}_{2}$ exposures in the two cohorts were quite different being $39.1 \mu \mathrm{g} / \mathrm{m}^{3}$ for the INMA cohort and only $13.6 \mu \mathrm{g} / \mathrm{m}^{3}$ for the MoBa cohort, in this case largely below the EU air quality standard of $40 \mu \mathrm{g} / \mathrm{m}^{3}$. The fact that wheezing incidence in early childhood was not associated with in utero exposure to traffic related air pollutants, whereas asthma incidence at school age was, allows several considerations: the lack of large studies and hence a problem of potency, the fact that wheezing in childhood and asthma are different disease entities or latency in disease manifestation.

There is little doubt on the relationship between acute exposure to high levels of air pollution and increased respiratory symptoms in children, including cough and wheeze, and visits to emergency departments for respiratory illnesses [7]. Whether there is also an association between early postnatal exposure to air pollution and wheezing and asthma development is a more contentious issue. In our systematic review an association between exposure to gases, in particular to $\mathrm{NO}_{2}$, but also in a number of studies to $\mathrm{PM}$, in particular to $\mathrm{PM}_{2.5}$, and asthma incidence has been reported in most of the studies.

In their systematic review and metanalysis, Bowatte and colleagues [8] concluded that exposure to trafficrelated air pollutants $\left(\mathrm{NO}_{2}, \mathrm{PM}_{2.5}\right.$, and $\left.\mathrm{BC}\right)$ from birth up to 5 years of age was associated with new onset of asthma throughout childhood. The association found between exposure to $\mathrm{NO}_{2}$ in the five studies meta-analysed was modest (OR 1.09; 95\% CI 0.96 to 1.23 per $10 \mathrm{mcg} /$ $\mathrm{m}^{3}$ increase) with a high heterogeneity between the studies. Association between $\mathrm{PM}_{2.5}$ (four studies) and $\mathrm{BC}$ (only three studies) and asthma incidence was slightly higher with an OR 1.14 (95\% CI 1.00 to 1.30) per $2 \mu \mathrm{g} /$ $\mathrm{m}^{3}$ increase and OR 1.20 (95\% CI 1.05 to 1.38$)$ per $1 \times$ $10^{-5} \mathrm{~m}^{-1}$ increase, respectively. Only few studies in the review of Bowatte and colleagues are included also in the present study, the others being on selected cohorts or evaluating exposure to pollutants beyond the first 2 years of children's life, raising a problem of overlap between the period of exposure measurement and that of outcome development. Among the more recent studies in our review (Tables 3 and 4 and supplementary Table 3), the association is expressed per one IQR increase of the air pollutants and a formal comparison among these studies and the older ones is difficult. Other methodological issues that could affect comparability among studies in our review are exposure models and age at outcome measurement. While more recent 
studies used models based on satellite data [12, 14-16, $18,19,28]$, allowing to obtain daily data and hence reliable exposure estimates in the first one or 2 years of life, studies published before 2015 mostly considered an average annual exposure estimated at the birth address. Furthermore, in most of these studies, exposure models based on air pollution measurements taken in different sampling campaigns done during several periods of one/ two weeks and then averaged to represent annual mean were used to assess exposure to air pollution at the birth address, and this could represent a problem for the assessment of a narrow exposure period like the first one or 2 years of life.

As for children age at asthma diagnosis, a study by Gehring et al. [29] aimed at assessing the longitudinal associations between exposure to air pollution and development of asthma, noticed that the effects of air pollution on asthma incidence were larger after the age of 4 years, where asthma diagnosis is more likely to be made. Though most of the studies in our review evaluating the association between air pollution exposure in the first 2 years of life and asthma incidence followed children up to school age, in some [14-21, 23, 27-34] a follow-up and hence asthma diagnosis was limited to the first years of life in all or in part of the subjects studied. This resulted also in a partial overlapping between the period of exposure and the development of the outcome. As already discussed, this overlap is more critical for studies that evaluated wheezing as an outcome. Interestingly, in two [22, 24] of the three studies [22-24] that evaluated wheezing phenotypes, there was an association between exposure to NOx and persistent wheezing at 4 years of life, a condition often associated with asthma.

\section{Conclusions}

Traffic-related air pollution during pregnancy increases the risk of asthma development among children and adolescents. This is in line with studies that considered lung function as an outcome. Also, in line with part of the studies on lung function is the finding of a susceptible time-window in the second trimester of pregnancy which corresponds to a period of intense airways development. We also confirmed a relationship between exposure in the first 2 years of life and asthma, although the time frame and hence the relationship between air pollutants exposure and asthma incidence needs to be further confirmed in studies with more precise exposure assessment. This is crucial for setting up more efficacious preventive strategies. Few studies with inconsistent results are available on the relationship between exposure to air pollutants either in pregnancy or in the 2 years after birth and wheezing development.

\section{Abbreviations}

BC: Black Carbon; Cl: Confidence Intervals; CO: Carbon Monoxide; EC: Elemental Carbon; ECAT: Elemental Carbon Attributed To Traffic; IQR: Interquartile Range; LUR: Land Use Regression; NO: Nitric Oxide; $\mathrm{NO}_{2}$ : Nitrogen Dioxide; NOx: Nitric Oxides; $\mathrm{O}_{3}$ : Ozone; PM: Particulate Matter; $\mathrm{SO}_{2}$ : Sulphur Dioxide; UFPS: Ultra-Fine Particles

\section{Supplementary Information}

The online version contains supplementary material available at https://doi. org/10.1186/s12940-021-00728-9.

Additional file 1: Supplementary Tables.

\section{Acknowledgements}

The authors are grateful to Dr. Salvatore De Masi (Meyer Children's University Hospital, Florence, Italy; Careggi University Hospital, Florence, Italy) for his thoughtful advice in conceiving the study.

\section{Authors' contributions}

FR conceived of the study and oversaw the analysis. AB and EG conducted the literature review. $A B, E G$ and $E M$ screened the literature records and assessed studies for eligibility. $A B$ and $E M$ extracted relevant data from the included studies and evaluated the risk of bias. FA and FR reviewed the extracted data and contributed to data interpretation. FR wrote the manuscript; $A B, E G, E M$ and $F A$ contributed to the manuscript writing. The authors read and approved the final manuscript.

\section{Funding}

The project was realized with the financial support of the Italian Ministry of Health - CCM.

Availability of data and materials

All data are available within the article and supplemental material.

\section{Declarations}

Ethics approval and consent to participate Not applicable.

Consent for publication

Not applicable.

Competing interests

The authors declare that they have no competing interests.

\section{Author details}

${ }^{1}$ Department of Experimental and Clinical Medicine, University of Florence, Florence, Italy. ${ }^{2}$ Coordinating Centre for Paediatric Rare Diseases, Meyer Children's University Hospital, Florence, Italy. ${ }^{3}$ Azienda Sanitaria Locale (ASL) TO 4, Ivrea (Turin), Italy. ${ }^{4}$ Department of Epidemiology, Lazio Regional Health Service, ASL Roma 1, Rome, Italy. ${ }^{5}$ Unit of Epidemiology, Meyer Children's University Hospital, Viale Pieraccini 24, 50139 Florence, Italy.

Received: 4 February 2021 Accepted: 12 April 2021

Published online: 17 April 2021

\section{References}

1. Darling JC, Bamidis PD, Burberry J, Rudolf MCJ. The first thousand days: early, integrated and evidence-based approaches to improving child health: coming to a population near you? Arch Dis Child. 2020;105(9):837-41. https://doi.org/10.1136/archdischild-2019-316929.

2. Schwarzenberg SJ, Georgieff MK, Committee On Nutrition. Advocacy for improving nutrition in the first 1000 days to support childhood development and adult health. Pediatrics. 2018;141(2):e20173716. https:// doi.org/10.1542/peds.2017-3716.

3. Wachs TD, Georgieff M, Cusick S, McEwen BS. Issues in the timing of integrated early interventions: contributions from nutrition, neuroscience, and psychological research. Ann N Y Acad Sci. 2014;1308(1):89-106. https:// doi.org/10.1111/nyas.12314. 
4. English R, Peer N, Honikman S, Tugendhaft A, Hofman KJ. 'First 1000 days' health interventions in low- and middle-income countries: alignment of South African policies with high-quality evidence. Glob Health Action. 2017;10(1):1340396.

5. McEvoy CT, Spindel ER. Pulmonary effects of maternal smoking on the fetus and child: effects on lung development, respiratory morbidities, and life long lung health. Paediatr Respir Rev. 2017;21:27-33. https://doi.org/10.101 6/j.prrv.2016.08.005.

6. Silvestri M, Franchi S, Pistorio A, Petecchia A, Rusconi F. Smoke exposure, wheezing, and asthma development: a systematic review and meta-analysis in unselected birth cohorts. Pediatr Pulmonol. 2015;50(4):353-62. https://doi. org/10.1002/ppul.23037.

7. Royal College of Physicians. Every breath we take: the lifelong impact of air pollution, report of a working party. London: Report of a working party, RCP; 2016. https://www.rcplondon.ac.uk/projects/outputs/everybreath-we-ta ke-lifelong-impact-air-pollution. Accessed 20 Apr 2020

8. Bowatte G, Lodge C, Lowe AJ, Erbas B, Perret J, Abramson MJ, et al. The influence of childhood traffic-related air pollution exposure on asthma, allergy and sensitization: a systematic review and meta-analysis of birth cohort studies. Allergy. 2015;70(3):245-56. https://doi.org/10.1111/all.12561.

9. Wells GA, Shea B, O'Connell D, et al. The Newcastle-Ottawa Scale (NOS) for Assessing the Quality of Nonrandomised Studies in Meta-analyses. Ottawa: Ottawa Hospital Research Institute. http://www.ohri.ca/programs/clinical_ epidemiology/oxford.asp. Accessed 17 Mar 2021

10. Soh S-E, Goh A, Teoh OH, Godfrey KM, Gluckman PD, Shek LP-C, et al. Pregnancy trimester-specific exposure to ambient air pollution and child respiratory health outcomes in the first 2 years of life: effect modification by maternal pre-pregnancy BMI. Int J Environ Res Public Health. 2018;15(5):996. https://doi.org/10.3390/ijerph15050996.

11. Madsen C, Haberg SE, Magnus MC, Aamodt G, Stigum H, London SJ, et al. Pregnancy exposure to air pollution and early childhood respiratory health in the Norwegian mother and child cohort study (MoBa). BMJ Open. 2017; 7(12):e015796. https://doi.org/10.1136/bmjopen-2016-015796.

12. Rosa MJ, Just AC, Kloog I, Pantic I, Schnass L, Lee A, et al. Prenatal particulate matter exposure and wheeze in Mexican children: effect modification by prenatal psychosocial stress. Ann Allergy Asthma Immunol. 2017:119(3):232-7 e1. https://doi.org/10.1016/j.anai.2017.06.016.

13. Aguilera I, Pedersen M, Garcia-Esteban R. Early-life exposure to outdoor air pollution and respiratory health, ear infections and eczema in infants from the INMA study. Environ Health Perspect. 2013;121(3):387-92. https://doi. org/10.1289/ehp.1205281.

14. Lavigne E, Donelle J, Hatzopoulou M, Van Ryswyk K, van Donkelaar A, Martin RV, et al. Spatiotemporal variations in ambient ultrafine particles and the incidence of childhood asthma. Am J Respir Crit Care Med. 2019; 199(12):1487-95. https://doi.org/10.1164/rccm.201810-19760C.

15. Jung CR, Chen WT, Tang YH, Hwang BF. Fine particulate matter exposure during pregnancy and infancy and incident asthma. J Allergy Clin Immunol. 2019;143(6):2254-2262.e5.

16. Lavigne É, Bélair MA, Rodriguez Duque D, Do MT, Stieb DM, Hystad P, et al. Effect modification of perinatal exposure to air pollution and childhood asthma incidence. Eur Respir J. 2018;51(3):1701884. https://doi.org/10.11 83/13993003.01884-2017.

17. Pennington AF, Strickland MJ, Klein M, Zhai X, Bates JT, Drews-Botsch C, et al. Exposure to Mobile source air pollution in early-life and childhood asthma incidence: the Kaiser air pollution and pediatric asthma study. Epidemiology. 2018;29(1):22-30. https://doi.org/10.1097/EDE.0000000000000754.

18. Lee A, Leon Hsu HH, Mathilda Chiu YH, Bose S, Rosa MJ, Kloog I, et al. Prenatal fine particulate exposure and early childhood asthma: effect of maternal stress and fetal sex. J Allergy Clin Immunol. 2018;141(5):1880-6. https://doi.org/10.1016/j.jaci.2017.07.017

19. Bose S, Chiu Y-HM, Hsu H-H L, Di Q, Rosa MJ, Lee A, et al. Prenatal nitrate exposure and childhood asthma. Influence of maternal prenatal stress and fetal sex. Am J Respir Crit Care Med. 2017;196(11):1396-403. https://doi. org/10.1164/rccm.201702-04210C.

20. Sbihi $H$, Koehoorn M, Tamburic L, Brauer M. Asthma trajectories in a population-based birth cohort. Am J Respir Crit Care Med. 2017;195(5):60713. https://doi.org/10.1164/rccm.201601-01640C.

21. Sbihi H, Tamburic $L$, Koehoorn M, Brauer M. Perinatal air pollution exposure and development of asthma from birth to age 10 years. Eur Respir J. 2016; 47(4):1062-71. https://doi.org/10.1183/13993003.00746-2015.

22. Rancière F, Bougas N, Viola M, Momas I. Early exposure to traffic-related air pollution, respiratory symptoms at 4 years of age, and potential effect modification by parental allergy, stressful family events, and sex: a prospective follow-up study of the PARIS birth cohort. Environ Health Perspect. 2017;125(4):737-45. https://doi.org/10.1289/EHP239.

23. Gehring $U$, Wijga $A H$, Brauer $M$, Fischer $P$, de Jongste JC, Kerkhof $M$, et al. Traffic-related air pollution and the development of asthma and allergies during the first 8 years of life. Am J Respir Crit Care Med. 2010;181(6):596603. https://doi.org/10.1164/rccm.200906-08580C.

24. Nordling E, Berglind N, MelMn E, Emenius G, Hallberg J, Nyberg F, et al. Traffic-related air pollution and childhood respiratory symptoms, Function and Allergies. Epidemiology. 2008;19(3):401-8. https://doi.org/10.1097/EDE. Ob013e31816a1ce3.

25. Morgenstern V, Zutavern A, Cyrys J, Brockow I, Gehring U, Koletzko S, et al. Respiratory health and individual estimated exposure to traffic-related air pollutants in a cohort of young children. Occup Environ Med. 2007;64(1):816. https://doi.org/10.1136/oem.2006.028241.

26. Brauer M, Hoek G, Van Vliet P, Meliefste K, Fischer PH, Wijga A, et al. Air pollution from traffic and the development of respiratory infections and asthmatic and allergic symptoms in children. Am J Respir Crit Care Med. 2002;166(8):1092-8. https://doi.org/10.1164/rccm.200108-0070C.

27. To T, Zhu J, Stieb D, et al. Early life exposure to air pollution and incidence of childhood asthma, allergic rhinitis and eczema. Eur Respir J. 2020;55(2): 1900913. https://doi.org/10.1183/13993003.00913-2019.

28. Tétreault LF, Doucet M, Gamache P, Fournier M, Brand A, Kosatsky T, et al. Childhood exposure to ambient air pollutants and the onset of asthma: an administrative cohort study in Québec. Environ Health Perspect. 2016;124(8): 1276-82. https://doi.org/10.1289/ehp.1509838.

29. Gehring U, Wijga AH, Hoek G, Bellander T, Berdel D, Brüske I, et al. Exposure to air pollution and development of asthma and rhinoconjunctivitis throughout childhood and adolescence: a population-based birth cohort study. Lancet Respir Med. 2015;3(12):933-42. https://doi.org/10.1016/S2213-2600(15)00426-9.

30. Gehring U, Beelen R, Eeftens M, Hoek G, de Hoogh K, de Jongste JC, et al. Wang $M$, smith HA, Brunekreef $B$. particulate matter composition and respiratory health. The PIAMA birth cohort study. Epidemiology. 2015;26(3): 300-9. https://doi.org/10.1097/EDE.0000000000000264.

31. Ranzi A, Porta D, Badaloni C, Cesaroni G, Lauriola P, Davoli M, et al. Exposure to air pollution and respiratory symptoms during the first 7 years of life in an Italian birth cohort. OccupEnviron Med. 2014;71:430-6.

32. Fuertes E, Standl M, Cyrys J, Berdel D, von Berg A, Bauer C-P, et al. A longitudinal analysis of associations between traffic-related air pollution with asthma, allergies and sensitization in the GINIplus and LISAplus birth cohorts. PeerJ. 2013;1:e193. https://doi.org/10.7717/peerj.193.

33. Gruzieva O, Bergström A, Hulchiy O, Kull I, Lind T, Melén E, et al. Exposure to air pollution from traffic and childhood asthma until 12 years of age. Epidemiology. 2013;24(1):54-61. https://doi.org/10.1097/EDE.0b013e318276clea.

34. Lindgren A, Stroh E, Björk J, Jakobsson K. Asthma incidence in children growing up close to traffic: a registry-based birth cohort. Environ Health. 2013;12(1):91. https://doi.org/10.1186/1476-069X-12-91.

35. Clark NA, Demers PA, Karr C, Koehoorn M, Lencar C, Tamburic L, et al. Effect of early life exposure to air pollution on development of childhood asthma. Environ Health Perspect. 2010;118(2):284-90. https://doi.org/10.1289/ehp.0900916.

36. Korten I, Ramsey K, Latzin P. Air pollution during pregnancy and lung development in the child. Paediatr Respir Rev. 2017;21:38-46. https://doi. org/10.1016/j.prrv.2016.08.008.

37. Cai Y, Hansell AL, Granell R, Blangiardo M, Zottoli M, Fecht D, et al. Prenatal, early-life, and childhood exposure to air pollution and lung function: the ALSPAC cohort. Am J Respir Crit Care Med. 2020;202(1):112-23. https://doi. org/10.1164/rccm.201902-02860C.

38. Bougas N, Rancière F, Beydon N, Viola M, Perrot X, Gabet S, et al. Trafficrelated air pollution, lung function, and host vulnerability. New insights from the PARIS birth cohort. Ann Am Thorac Soc. 2018;15(5):599-607. https://doi.org/10.1513/AnnalsATS.201711-9000C.

39. Morales E, Garcia-Esteban R, Asensio de la Cruz O, et al. Intrauterine and early postnatal exposure to outdoor air pollution and lung function at preschool age. Thorax. 2015;70(1):64-73. https://doi.org/10.1136/thoraxjnl-2014-205413.

40. Rice MB, Mein SA. Prenatal air pollution and child lung function: the impossible search for a vulnerable trimester. Am J Respir Crit Care Med. 2020;202(1):15-6. https://doi.org/10.1164/rccm.202003-0764ED.

\section{Publisher's Note}

Springer Nature remains neutral with regard to jurisdictional claims in published maps and institutional affiliations. 